\title{
Influence of radiation exposure pattern on the bone injury and osteoclastogenesis in a rat model
}

\author{
JIANGLONG ZHAI, FEILONG HE, JIANPING WANG, JUNXIANG CHEN, LING TONG and GUOYING ZHU \\ Department of Radiation Protection, Institute of Radiation Medicine, Fudan University, Shanghai 200032, P.R. China
}

Received May 8, 2019; Accepted September 20, 2019

DOI: $10.3892 /$ ijmm.2019.4369

\begin{abstract}
Radiotherapy, one of the clinical treatments of cancer, is accompanied by a high risk of damage to healthy tissues, such as bone loss and increased risk of fractures. The aim of the present study was to establish a rat model of local and systemic bone injury by focal irradiation, in order to study the etiological mechanism and intervention. The proximal metaphyseal region of the left hindlimb of male Sprague-Dawley rats were exposed to a single $2 \mathrm{~Gy}$ or three $8 \mathrm{~Gy}$ doses delivered on days 1, 3 and 5 using a small animal irradiator, the changes in bone volume and microarchitecture were evaluated, and the mineral apposition rate (MAR) was assessed. Furthermore, bone marrow-derived macrophages (BMMs) were isolated and induced to osteoclasts. It has been demonstrated that a single dose of 2 Gy may result in a significant loss of lumbar bone density at 3 days post-irradiation, however this is restored at 30 days post-irradiation. In the $3 \times 8$ Gy irradiation rat model, there was a rapid decrease in the aBMD of lumbar spine at 3 days and at 7 days post-irradiation, and the aBMD decline persisted even at 60 days post-irradiation. In addition, microCT analysis revealed a persistent decline in bone volume and damage in microarchitecture in the $3 \times 8$ Gy irradiation model, accompanied by a decrease in MAR, index of the decline in bone-forming ability. In the cellular mechanism, a single $2 \mathrm{~Gy}$ local irradiation mainly manifested as an enhancement of the BMMs osteoclastogenesis potential, which was different from the osteoclastogenesis inhibition after high-dose focal irradiation (3x8 Gy). In summary, the irradiation with simulated clinical focal fractionated radiotherapy exerts short- and long-term systemic injury on bone tissue, characterized by different osteoclastogenesis potential between the high dose mode and a single 2 Gy focal irradiation. Physicians must consider the irreversibility of bone damage in clinical radiotherapy.
\end{abstract}

Correspondence to: Mrs. Guoying Zhu, Department of Radiation Protection, Institute of Radiation Medicine, Fudan University, 2094 Xietu Road, Shanghai 200032, P.R. China

E-mail: zhugy@shmu.edu.cn

Key words: local irradiation, bone injury, mineral apposition, osteoclast, rat model

\section{Introduction}

Cancer treatment, including radiotherapy (RT), may adversely affect bone health (1-3). In addition to bone-related events associated with tumor bone metastasis, cancer treatment per se may also lead to bone injury, termed cancer treatment-induced bone loss (CTIBL), resulting in non-tumor bone pain, bone atrophy and susceptibility to pathological fractures $(4,5)$. The long-term side effects on bone caused by anti-cancer treatment have attracted a large amount of attention $(6,7)$. RT is an effective and valuable method for anti-cancer treatment, as $>50 \%$ of cancer patients require RT, alone or in combination with chemotherapy, surgery and other therapeutic methods, at some stage of the malignancy $(8,9)$. The radiation dose to the target tissue may reach 50-90 Gy, and normal tissues surrounding the target area, including bone tissue, may also absorb ionizing radiation $(8,9)$. It has been demonstrated that pelvic irradiation significantly increases the risk of female pelvic fracture $(4,5)$, and rib fractures often occur following external stereotactic radiotherapy for lung cancer (10). Additionally, evidence on radiation-induced local and systemic bone loss has also been demonstrated in local or total body irradiated mouse models (11-13). RT may pose a risk to skeletal health, leading to bone reconstruction imbalance and bone loss in the exposed areas (local bone loss), as well as the remote unexposed areas (systemic bone loss), and may aggravate the risk of cancer recurrence and bone metastasis due to the disintegration of bone matrix and release of active factors $(14,15)$. Irradiation acutely stimulates bone remodeling in mice; however, the long-term influence of irradiation on bone-resorbing potential (osteoclastogenesis) and the possible adaptive mechanisms are not well understood, which is important for developing prevention and treatment strategies for malignancies.

RT-induced bone loss has become an important factor limiting the effective therapeutic dose, affecting the quality of life and prognosis of patients $(16,17)$. One of the important events in tumor RT is the choice of dosage and fractionation mode (18-20). Single 2 Gy irradiation has often been used in animal models (21), although this is only equivalent to the clinical RT routine split single dose and astronaut space flight dose, which are far lower than the total dose in the clinical radiotherapy. In addition, the choice of irradiation site can also affect the radiation effects, while total body irradiation may cause a systemic inflammatory response and may even lead to hypogonadism, complicating the interpretation of 
experimental data related to bone injury (22). In the present study, a rat model of radiation-induced bone loss was constructed to simulate clinical focal fractionated RT, which may be used to investigate the effects of ionizing radiation on bone structure, bone tissue cells and other biological processes in vivo, and may help elucidate the etiological mechanism of bone loss following RT.

\section{Materials and methods}

Animals and irradiation. Male Sprague-Dawley rats (age, 6 weeks; weight 80-90 g; $n=120$ ), were obtained from the Department of Experimental Animal of Fudan University (Shanghai, China). All animal experimental procedures were approved by the Committee for Ethical Use of Experimental Animal at Fudan University (ethical approval registration no. 2017-03-FYS-ZGY-01). The rats were randomized into the following groups: i) control group for the 2 Gy model; ii) single local irradiation ( $2 \mathrm{~Gy}$ ) group; iii) control group for the (3x8 Gy) model; and iv) local fractionated irradiation ( $3 \times 8 \mathrm{~Gy})$ group. There were 6 rats in each group at each time point. The rats were locally irradiated in the left hindlimb with a single 2 Gy or three 8 Gy doses delivered on days 1, 3 and 5, using the X-RAD 320 Biological Irradiator (X-RAD 320, PXi) with a collimator (diameter, $1.8 \mathrm{~cm}$; covering areas of the left femur and tibia) or a self-made shielding device made up of 8-mm thick lead. The dose rate was $185.5 \mathrm{cGy} / \mathrm{min}$, with $300 \mathrm{kV}$ tube voltage, $12 \mathrm{~mA}$ tube current, and a distance of $60 \mathrm{~cm}$ between the source and the surface. $\mathrm{LiF}(\mathrm{Mg}, \mathrm{Cu}, \mathrm{P})$ thermoluminescent dosimeters were used to measure the actual radiation dose at different distances from the radiation center $(23,24)$. The rats were anesthetized with intraperitoneal injection of $1 \%$ sodium pentobarbital (40 mg/kg), fixed on the anatomical plate in the supine position, the left hindlimb was stretched outside the shielding lead box, and the remaining body parts were positioned inside the shielding lead box. The rats and the shielding lead box were placed into the irradiator, and the rats of the local radiation group were exposed to X-ray irradiation, whereas the rats of the control group received no irradiation. During the experimental period, the environmental temperature was $20-26^{\circ} \mathrm{C}$, with a light/dark cycle of 12/12 h, and the rats were housed for 2 weeks of acclimatization and allowed free access to food and water.

Sample collection. The left hindlimb of the control group was defined as the 'control limb', the left hindlimb of the irradiated group as the 'local irradiated limb', and the right hindlimb as the 'contralateral limb'. At 3, 7, 14, 30 and 60 days after the first irradiation, 6 rats in each group were weighed and euthanized, followed by collection of blood samples and harvesting of the L1-L5 lumbar vertebrae, bilateral femora and tibiae. At the time points of 30 and 60 days, the rats were injected intraperitoneally with $5 \mathrm{mg} / \mathrm{kg}$ calcein (Shanghai Macklin Biochemical Co., Ltd.) and $30 \mathrm{mg} / \mathrm{kg}$ tetracycline hydrochloride (Shanghai Macklin Biochemical Co., Ltd.) for double fluorescence labeling on the 14th and 4th day prior to euthanasia, respectively. The protocol of local irradiation and sampling are presented in Fig. 1.

Measurement of areal bone mineral density (aBMD). The L1-L5 lumbar vertebrae were harvested, the surrounding connective tissue was trimmed, and dual-energy X-ray absorptiometry was used to measure aBMD (the instrument accuracy was $1 \%$, and the coefficient of variation was $<1 \%$ ).

Micro-computed tomography (microCT) analysis and $3 D$ reconstruction. The microCT scanning sites comprised the entire unilateral femur and L3 lumbar vertebra. Bone specimens were fixed in $10 \%$ formaldehyde for $48 \mathrm{~h}$ at $37^{\circ} \mathrm{C}$. The scanning resolution was $18 \mu \mathrm{m}$ under the following conditions: $80 \mathrm{kV} / 313 \mu \mathrm{A} / 250 \mathrm{msec}, 0.5 \mathrm{~mm}$ aluminum filter, $180^{\circ}$ spiral scan. The image data were reconstructed by NRecon software (version 1.6.9.8; Bruker), while the coronal, sagittal and transverse bone structure was observed by DataViewer software (version 1.5.1.2; Bruker). 3D analysis was performed by CTAn software (version 1.15.4.0+; Bruker), and the 3D rendering was created by CTvox software (version 2.6.0 r 908; Bruker). The region of interest (ROI) was selected according to the distal femur growth plate, and bone morphometric analysis of cancellous bone was conducted with CTAn software. The trabeculae at the distal end of the femur and the L3 lumbar vertebra were included in the cancellous bone analysis site. The ROI of the trabeculae and cortical in femur were evaluated with a region with a spacing of $1.00 \mathrm{~mm}$. The main parameters of cancellous bone analysis included volumetric BMD (vBMD), bone volume (BV)/tissue volume (TV), trabecular number (Tb.N), trabecular thickness (Tb.Th), trabecular separation (Tb.Sp) and rabecular bone pattern factor (Tb.Pf). Cortical bone thickness (Ct.Th) was the main analytical parameter of cortical bone.

Dualfluorescence labeling. The rats were administered calcein and tetracycline hydrochloride by intraperitoneal injection on the 14th and the 4th day prior to euthanasia. The tibiae were fixed in Million's Buffer containing $10 \%$ formaldehyde at $4^{\circ} \mathrm{C}$ for 3 days, washed with water overnight, dehydrated with 40, 70, 95 and $100 \%$ ethanol for 1 day each, soaked in xylene for 1 day, and embedded in methyl methacrylate. The bone specimens were cut into $20-\mu \mathrm{m}$ sections, observed and photographed using a fluorescence microscope (magnification, $\mathrm{x} 400$ ), and the distance between the double fluorescence labeled lines was measured to calculate the mineral apposition rate $(\mathrm{MAR})$ as follows: MAR $(\mu \mathrm{m} /$ day $)=$ double fluorescence line spacing/days between the two lines.

Osteoclastogenesis from bone marrow-derived macrophages $(B M M s)$. BMMs were isolated from the bone marrow of experimental rats and induced to differentiate into osteoclasts in the presence of $40 \mathrm{ng} / \mathrm{ml}$ receptor activator of nuclear factor- $\kappa \mathrm{B}$ ligand (RANKL; PeproTech) and $25 \mathrm{ng} / \mathrm{ml}$ macrophage colony-stimulating factor (M-CSF; PeproTech) at $37^{\circ} \mathrm{C}$. The cells were cultured in $\alpha$-minimal essential medium (Gibco; Thermo Fisher Scientific, Inc.) supplemented with 10\% FBS (Gibco; Thermo Fisher Scientific, Inc.) and 1\% penicillin/streptomycin (Gibco; Thermo Fisher Scientific, Inc.) at $37^{\circ} \mathrm{C}$ in a humidified incubator containing $5 \% \mathrm{CO}_{2}$. The induction medium was replenished every 2 days. After 7 days, the cells were fixed in $2.5 \%$ glutaraldehyde at $37^{\circ} \mathrm{C}$ for $5 \mathrm{~min}$ and stained for tartrate-resistant acid phosphatase (TRAP) activity at $37^{\circ} \mathrm{C}$ for $1 \mathrm{~h}$ by a TRAP staining kit (Sigma-Aldrich; Merck $\mathrm{KGaA}$ ). Images of cells were obtained using a fluorescence microscope (magnification, $\mathrm{x} 40$ ). TRAP-positive multinucle- 


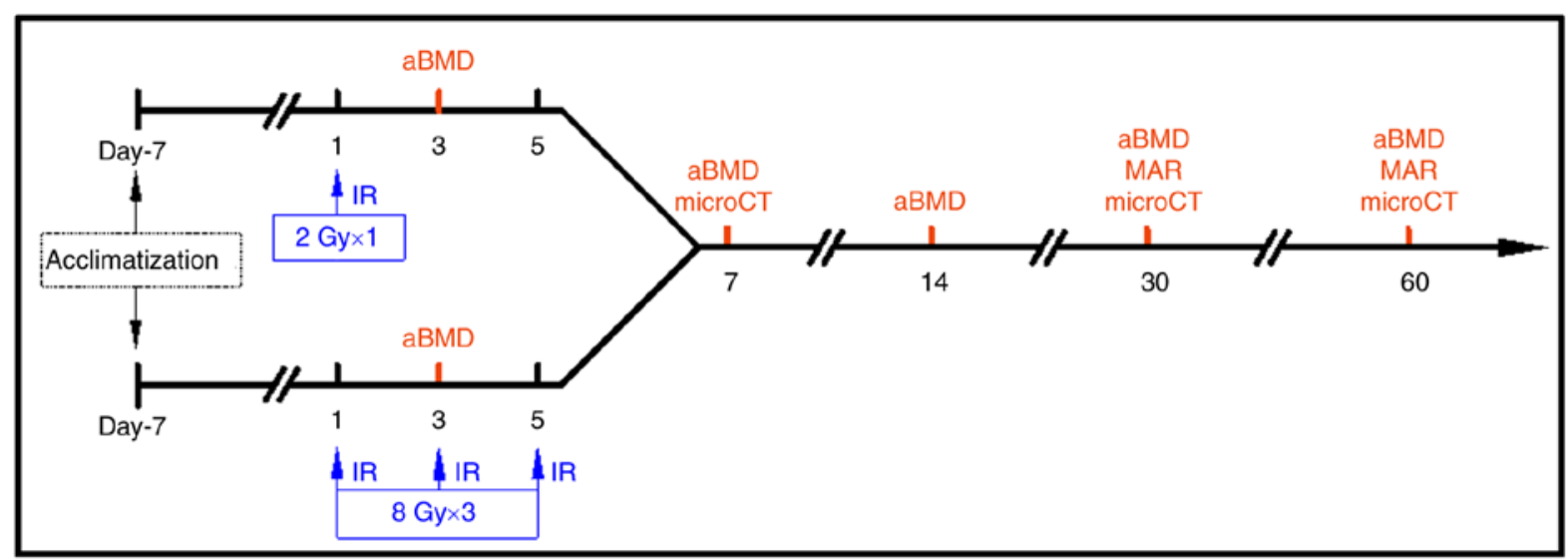

Figure 1. Time course of local irradiation experiment in rats. aBMD, areal bone mineral density; microCT, micro-computed tomography; IR, irradiation; MAR, mineral apposition rate.

ated cells with $>3$ nuclei were defined as osteoclasts, and the number of TRAP-positive cells was counted by Simple PCI software (version 5.2.1.1609; Compix, Inc.).

Reverse transcription-quantitative PCR (RT-qPCR). Total RNA from BMM-derived osteoclasts was extracted using RNAprep Pure Cell/Bacteria kit (Tiangen Biotech Co., Ltd.) and converted to complementary DNA by Quantscript RT kit (Tiangen Biotech Co., Ltd.) at $45^{\circ} \mathrm{C}$ for $15 \mathrm{~min}$ and $95^{\circ} \mathrm{C}$ for 3 min. qPCR reactions $(20 \mu \mathrm{l})$ were performed with SYBR Premix Ex Taq mix (Takara Bio, Inc.) with the following thermocycling conditions: $95^{\circ} \mathrm{C}$ for $30 \mathrm{sec}$, followed by 40 cycles of $95^{\circ} \mathrm{C}$ for $5 \mathrm{sec}, 60^{\circ} \mathrm{C}$ for $30 \mathrm{sec}$ and $72^{\circ} \mathrm{C}$ for $30 \mathrm{sec}$, and once cycle of $65^{\circ} \mathrm{C}$ for $15 \mathrm{sec}$. A qPCR system (Light Cycler 2.0; Roche Diagnostics $\mathrm{GmbH}$ ) was applied to determine the mRNA level. The primer sequences are listed in Table I. The $2^{-\Delta \Delta \mathrm{Cq}}$ method was used to calculate the relative mRNA expression (25).

Statistical analysis. Data are expressed as the mean \pm standard deviation, and were evaluated by one-way ANOVA using SPSS 16.0 software (SPSS, Inc.), followed by Bonferroni's correction for multiple comparisons. Each experiment was repeated a minimum of three times. $\mathrm{P}<0.05$ was considered to indicate a statistically significant difference.

\section{Results}

Quality assurance of local irradiation process. An X-ray irradiator and collimator were used in the present study. Considering the actual length of the rat hindlimb, a collimator with a diameter of $1.8 \mathrm{~cm}$ was used (covering most of the femur and tibia of the unilateral hindlimb), and the center of collimator coincided with the center of irradiated rat hind limbs. A $\mathrm{LiF}(\mathrm{Mg}, \mathrm{Cu}, \mathrm{P})$ thermoluminescent dosimeter was used to measure the actual radiation dose at different distances from the irradiated central point, in order to verify the central effect of the collimator. When the center of the hindlimb overlaps with the collimator, the dose rate at the edge of the collimator $\sim 1$ and $2.5 \mathrm{~cm}$ from the center decreases by 58.9 and $99.1 \%$, respectively. Therefore, considering the uniformity of dose distribution, the collimator was not adopted; a self-designed shielding device made up of 8-mm thick lead was used instead. Using this irradiation model, only the left hindlimb outside the device was exposed to X-rays with $\sim 2.0 \mathrm{~cm}$ diameter, which was equivalent to the bone volume of the locally irradiated pelvis in the clinical setting. Using this irradiation equipment, the dose distribution within the radiation field was ideally uniform, while the dosage inside the lead box could be reduced by $>99.5 \%$.

Physical conditions of irradiated rats. All experimental rats survived during the study period. Following single 2 Gy or $3 \times 8$ Gy fractionated local irradiation, the rat weight in the irradiated group was significantly lower than that in control group at 3 days post-irradiation $(\mathrm{P}<0.05)$; however, the early loss of weight could be gradually restored (Fig. 2). Irradiation exposure simulating the clinical local RT exerted no long-term effects on the physical status.

Effects of local irradiation on aBMD. LumbaraBMD decreased at 3 days after single 2 Gy or 3x8 Gy local irradiation, and the differences were statistically significant compared with the control groups $(\mathrm{P}<0.05)$. The lumbar vertebral density of rats locally irradiated with 2 Gy recovered to a certain extent, and the lumbar aBMD exhibited no significant change at day 60 . However, in the $3 \times 8$ Gy irradiation model, the lumbar aBMD of locally exposed rats was significantly lower at day 7 $(\mathrm{P}<0.05)$, which was 2 days after the third irradiation (total dose, 24 Gy). There was no obvious recovery at 2 months after radiation exposure (Table II). As aforementioned, compared with the conventional single 2 Gy irradiation model, the bone loss in the $3 \times 8$ Gy local irradiation model, which mimics clinical fractionated RT, occurred at an earlier stage and was difficult to restore.

MicroCT analysis of bone morphometric parameters in locally irradiated rats. The femora and lumbar vertebrae of locally irradiated rats were reconstructed by microCT scan, and the local and systemic effects of local radiation exposure to the left hindlimb were analyzed at different time points after radiation exposure.

First, in the single 2 Gy local irradiation model, microCT imaging revealed deteriorated trabecular and cortical micro- 
Table I. Primer sequences for reverse transcription-quantitative PCR.

\begin{tabular}{lll} 
Target gene & \multicolumn{1}{c}{ Forward (5'-3') } & \multicolumn{1}{c}{ Reverse (5'-3') } \\
\hline GAPDH & CTGCACCACCAACTGCTTAG & AGATCCACGACGGACACATT \\
Cathepsin K & ACGGAGGCATCGACTCTGAA & GATGCCAAGCTTGCGTCGAT \\
Integrin $\beta 3$ & ATTGAGTTCCCAGTCAGTGAG & GACAGGTCCATCAAGTAGTAG
\end{tabular}

Table II. Effects of local irradiation on $\mathrm{aBMD}\left(\mathrm{g} / \mathrm{cm}^{2}\right)$ in lumbar spine of rats $(\mathrm{n}=6)$.

\begin{tabular}{lccccc}
\hline & \multicolumn{2}{c}{ Single local irradiation $(2 \mathrm{~Gy})$} & & \multicolumn{2}{c}{ Fractionated local irradiation (3x8 Gy) } \\
\cline { 2 - 3 } \cline { 5 - 6 } Days post-irradiation & Control group & Local irradiated group & & Control group & Local irradiated group \\
\hline 3 & $0.116 \pm 0.007$ & $0.096 \pm 0.005^{\mathrm{a}}$ & & $0.185 \pm 0.017$ & $0.161 \pm 0.007^{\mathrm{a}}$ \\
7 & $0.122 \pm 0.011$ & $0.124 \pm 0.007$ & & $0.195 \pm 0.007$ & $0.182 \pm 0.007^{\mathrm{a}}$ \\
14 & $0.156 \pm 0.006$ & $0.149 \pm 0.008$ & & $0.217 \pm 0.010$ & $0.213 \pm 0.010$ \\
30 & $0.218 \pm 0.005$ & $0.217 \pm 0.016$ & & $0.270 \pm 0.009$ & $0.268 \pm 0.028$ \\
60 & $0.323 \pm 0.020$ & $0.315 \pm 0.009$ & & $0.330 \pm 0.022$ & $0.299 \pm 0.025^{\mathrm{a}}$ \\
\hline
\end{tabular}

${ }^{\mathrm{a}} \mathrm{P}<0.05$ vs. control group. aBMD, areal bone mineral density.
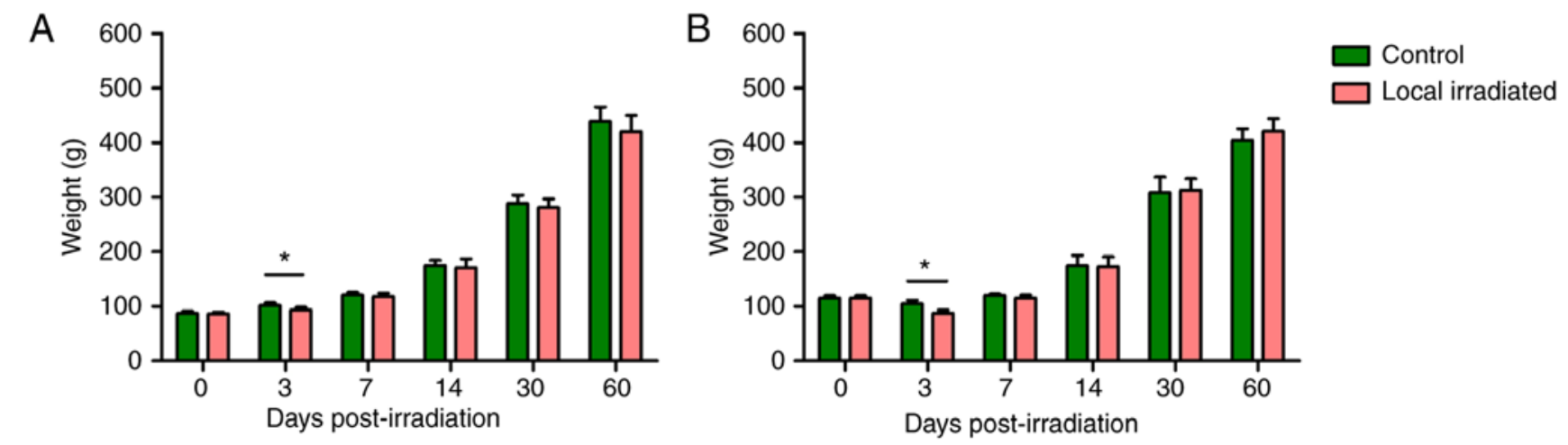

Figure 2. Weight change in rats after local irradiation. (A) Single 2 Gy local irradiation. (B) 3x8 Gy local irradiation. The weight of irradiated rats was significantly lower compared with that of the control rats at 3 days post-irradiation exposure; however, the early weight loss was gradually restored, and there was no significant difference between the two groups at 7 to 60 days of radiation exposure. $n=6$. ${ }^{*} \mathrm{P}<0.05$.

architecture in both the directly irradiated and contralateral femora, as early as 7 days post-irradiation. The femoral bone was obviously sparse and brittle, with a significant reduction in vBMD, BV/TV fraction, Tb.Th, Tb.N and Ct.Th, accompanied by an increase in Tb.Pf. However, at 30 days post-irradiation, the changes in bone morphometric parameters of the locally irradiated and the contralateral hindlimb had recovered well (Fig. 3; Table III). Therefore, after a single 2 Gy local irradiation, early transient bone damage may occur in both the irradiated and contralateral femur, suggesting that there is a systemic effect of bone irradiation, albeit reversible.

The lumbar microCT scan also demonstrated a significant decrease in the vBMD, BV/TV and Tb.Th, and an increase in Tb.Pf in rats exposed to a single 2 Gy local irradiation at 7 days post-irradiation, but the bone damage in the lumbar spine was restored at 30 days post-irradiation, and a similar trend was observed in the femoral bone (Fig. 3; Table IV).

Second, in the $3 \times 8$ Gy local irradiation model, the trabecular bone was obviously sparse and brittle, with significant bone loss and bone structural damage in both the directly irradiated and the contralateral hindlimbs as early as 7 days post-irradiation. A decrease in vBMD, BV/TV and Tb.N was observed in the directly irradiated group, while in the contralateral hindlimbs there was a decrease in vBMD and an increase in Tb.Sp. Furthermore, the bone loss and structural damage in the directly irradiated femoral bone persisted at 60 days post-irradiation (Fig. 4; Table V), with a decrease in vBMD, BV/TV and Tb.N, accompanied by an increase in Tb.Sp. The results suggested that the fractioned local irradiation could induce early bone loss and long-term bone structural damage in the directly irradiated areas, but this was reversible in the indirectly irradiated areas due to the lower dose. 
Table III. Morphometric parameter values of femoral bone after single $2 \mathrm{~Gy}$ local irradiation $(\mathrm{n}=6)$.

\begin{tabular}{|c|c|c|c|c|c|c|}
\hline \multirow[b]{2}{*}{$\begin{array}{l}\text { Morphometric } \\
\text { parameters }\end{array}$} & \multicolumn{3}{|c|}{7 days post-irradiation } & \multicolumn{3}{|c|}{30 days post-irradiation } \\
\hline & Control limb & $\begin{array}{c}\text { Contralateral } \\
\text { limb }\end{array}$ & $\begin{array}{l}\text { Local } \\
\text { irradiated limb }\end{array}$ & $\begin{array}{l}\text { Control } \\
\text { limb }\end{array}$ & $\begin{array}{l}\text { Contralateral } \\
\operatorname{limb}\end{array}$ & $\begin{array}{c}\text { Local } \\
\text { irradiated limb }\end{array}$ \\
\hline $\mathrm{vBMD}, \mathrm{g} / \mathrm{mm}^{3}$ & $0.268 \pm 0.010$ & $0.230 \pm 0.059$ & $0.240 \pm 0.008^{\mathrm{a}}$ & $0.227 \pm 0.016$ & $0.220 \pm 0.025$ & $0.224 \pm 0.010$ \\
\hline $\mathrm{BV} / \mathrm{TV}, \%$ & $23.34 \pm 4.700$ & $19.18 \pm 2.769$ & $15.03 \pm 1.096^{\mathrm{a}}$ & $33.17 \pm 4.283$ & $33.15 \pm 0.704$ & $33.49 \pm 3.055$ \\
\hline Tb.Th, mm & $0.112 \pm 0.003$ & $0.108 \pm 0.005$ & $0.102 \pm 0.004^{\mathrm{a}}$ & $0.117 \pm 0.004$ & $0.116 \pm 0.002$ & $0.119 \pm 0.002$ \\
\hline $\mathrm{Tb} . \mathrm{N}, 1 / \mathrm{mm}$ & $2.072 \pm 0.393$ & $1.784 \pm 0.346$ & $1.483 \pm 0.126^{\mathrm{a}}$ & $2.831 \pm 0.302$ & $2.720 \pm 0.101$ & $2.804 \pm 0.200$ \\
\hline Tb.Sp, mm & $0.396 \pm 0.136$ & $0.471 \pm 0.080$ & $0.483 \pm 0.095$ & $0.242 \pm 0.029$ & $0.245 \pm 0.015$ & $0.247 \pm 0.035$ \\
\hline Tb.Pf, $1 / \mathrm{mm}$ & $8.587 \pm 1.234$ & $10.90 \pm 1.047^{\mathrm{a}}$ & $12.95 \pm 1.219^{\mathrm{a}}$ & $4.259 \pm 1.943$ & $4.731 \pm 1.314$ & $4.405 \pm 0.627$ \\
\hline Ct.Th, mm & $0.277 \pm 0.024$ & $0.265 \pm 0.019$ & $0.252 \pm 0.010^{\mathrm{a}}$ & $0.469 \pm 0.013$ & $0.465 \pm 0.017$ & $0.470 \pm 0.022$ \\
\hline
\end{tabular}

${ }^{\mathrm{a}} \mathrm{P}<0.05$ control vs. irradiated or contralateral. vBMD, volume bone mineral density; BV/TV, bone volume fraction; Tb.Th, trabecular thickness; Tb.N, trabecular number; Tb.Sp, trabecular separation; Tb.Pf, trabecular pattern factor; Ct.Th, cortical bone thickness.

A
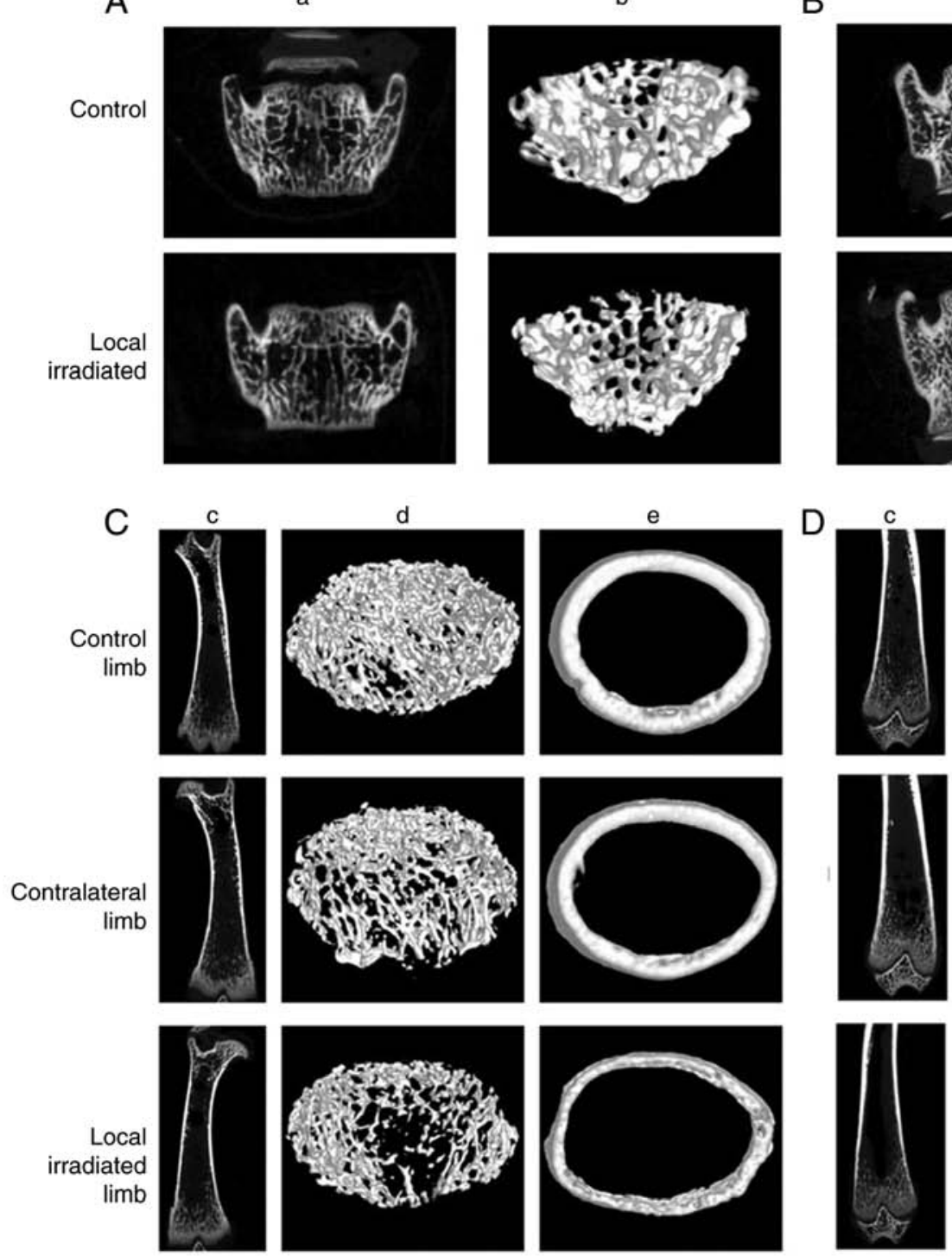

B
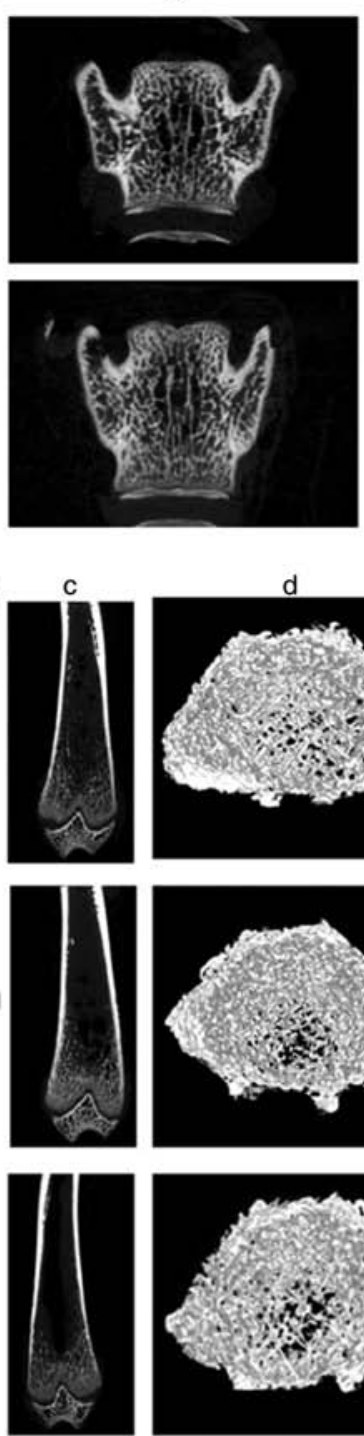
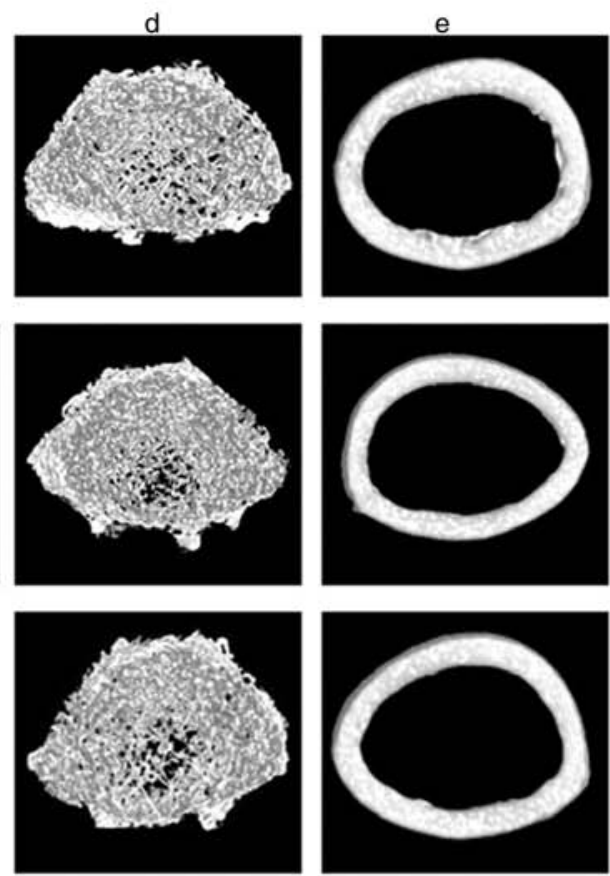

Figure 3. microCT reconstructions of femur and lumbar vertebra at 7 and 30 days after single 2 Gy local irradiation. Lumbar trabecular bone of the region of interest at (A) 7 and (B) 30 days post-irradiation. (a) Representative two-dimensional and (b) three-dimensional images of trabecular bone in lumbar spine. Femoral trabecular and cortical bone of the region of interest at (C) 7 and (D) 30 days post-irradiation. (c) Coronal position of femur (two-dimensional); (d) femoral trabecular bone (three-dimensional); and (e) femoral cortical bone (three-dimensional). microCT, micro-computed tomography. 
Table IV. Morphometric parameter values of lumbar vertebrae after single 2 Gy local irradiation $(n=6)$.

7 days post-irradiation

Morphometric parameters

vBMD, $\mathrm{g} / \mathrm{mm}^{3}$

$\mathrm{BV} / \mathrm{TV}, \%$

Tb.Th, mm

Tb.N, $1 / \mathrm{mm}$

Tb.Sp, $\mathrm{mm}$

Tb.Pf, $1 / \mathrm{mm}$
Local radiation group

Control group

$0.261 \pm 0.014$
$41.58 \pm 3.590$
$0.109 \pm 0.006$
$3.820 \pm 0.358$
$0.167 \pm 0.012$
$5.195 \pm 1.654$

$0.209 \pm 0.013^{\mathrm{a}}$

$36.89 \pm 2.831^{\mathrm{a}}$

$0.107 \pm 0.003^{\mathrm{a}}$

$3.444 \pm 0.261$

$0.179 \pm 0.011$

$6.988 \pm 0.894^{\mathrm{a}}$
30 days post-irradiation

\begin{tabular}{cc}
\hline $\begin{array}{c}\text { Control } \\
\text { group }\end{array}$ & $\begin{array}{c}\text { Local } \\
\text { radiation group }\end{array}$ \\
$0.266 \pm 0.028$ & $0.259 \pm 0.021$ \\
$59.43 \pm 4.416$ & $58.09 \pm 3.264$ \\
$0.130 \pm 0.005$ & $0.129 \pm 0.005$ \\
$4.559 \pm 0.223$ & $4.363 \pm 0.281$ \\
$0.120 \pm 0.009$ & $0.128 \pm 0.010$ \\
$6.005 \pm 2.076$ & $7.047 \pm 1.311$ \\
\hline
\end{tabular}

${ }^{\mathrm{a}} \mathrm{P}<0.05$ vs. control. vBMD, volume bone mineral density; BV/TV, bone volume fraction; Tb.Th, trabecular thickness; Tb.N, trabecular number; Tb.Sp, trabecular separation; Tb.Pf, trabecular pattern factor.
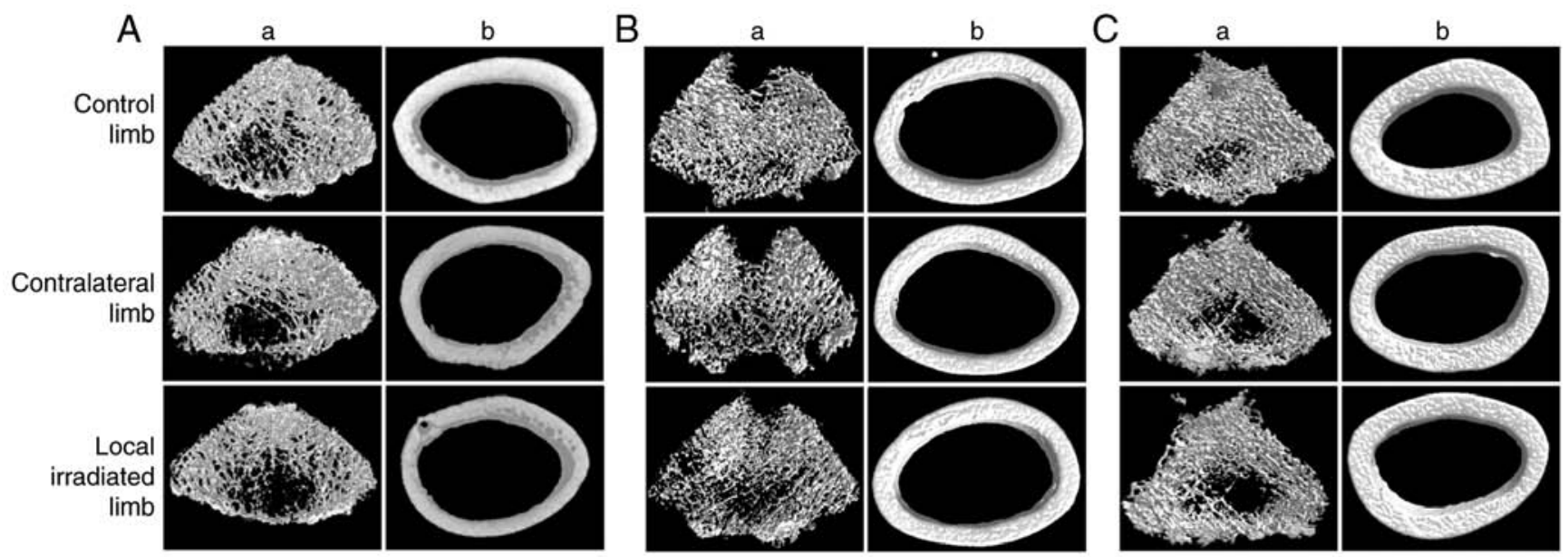

Figure 4. microCT reconstructions of femur at 7, 30 and 60 days after $3 \times 8$ Gy local irradiation. Femoral trabecular and cortical microarchitecture in the region of interest at (A) 7, (B) 30 and (C) 60 days post-irradiation. (a) Representative femoral trabecular bone (three-dimensional) and (b) femoral cortical bone (three-dimensional). microCT, micro-computed tomography.

Effect of local irradiation on bone-forming ability. The effects of different irradiation doses on the MAR of rats were determined by calcein and tetracycline hydrochloride double fluorescence labeling method. The results demonstrated that, at 30 and 60 days after single local irradiation with $2 \mathrm{~Gy}$, the MAR of the directly exposed hindlimbs and the contralateral hindlimbs did not differ significantly from that of control group rats, i.e., the single 2 Gy irradiation exerted no significant effect on the bone formation ability. However, at 30 days after irradiation with $3 \times 8$ Gy, MAR in the directly irradiated hindlimbs exhibited a significant decrease by $>50 \%$ compared with the control limbs. At 60 days after $3 \times 8$ Gy local irradiation, the MAR of the irradiated limb remained reduced by $31.1 \%$ compared with the control limb, but exhibited an obvious improvement compared with that at 30 days (Fig. 5).

Changes of osteoclast differentiation potential of locally irradiated BMMs in rats. BMMs were obtained at 7 and 30 days post-irradiation, induced and differentiated into osteoclasts. TRAP staining was performed, and TRAP-positive multinuclear cells were counted on day 7 . The results revealed that the number of TRAP-positive multinuclear cells from BMMs of the irradiated rats increased markedly compared with the control group, and the mRNA expression of levels cathepsin K and integrin 3, two well-known markers of osteoclast differentiation, were significantly upregulated at 7 days post-irradiation $(\mathrm{P}<0.05)$. However, at 30 days post-irradiation, the number of TRAP-positive multinucleated cells exhibited a decrease to normal, and the differentiation marker gene expression levels did not differ significantly compared with the control group ( $\mathrm{P}>0.05$; Fig. 6).

When 3x8 Gy local irradiation was applied, the ability of osteoclastogenesis of BMMs from both the directly exposed hindlimbs and the contralateral hindlimb at 7 days post-irradiation was significantly decreased compared with the control group, showing that the number of TRAP-positive multinuclear cells was significantly reduced $(\mathrm{P}<0.05)$. At 30 days post-irradiation, the osteoclastogenesis potential of BMMs returned to normal levels, with no statistically significant differences compared with the control group (Fig. 7). In summary, unlike the single 2 Gy irradiation model, the number of TRAP-positive multinuclear cells generated by 
BMM-induced fusion was significantly reduced in the simulated clinical 3x8 Gy fractionated irradiation model, even at the early post-irradiation stage, possibly due to the excessive inhibition of the bone marrow by high-dose irradiation.

\section{Discussion}

Currently, surgical treatment, chemotherapy and RT are the common methods of cancer treatment, and the curative effect of RT has been generally recognized. However, ionizing radiation exerts a damage effect on normal bone tissue. Evidence on radiation-induced bone loss has been widely reported in female patients with pelvic cancer and male patients with prostate cancer, and the appropriate RT dosage and the fractionation mode are key factors affecting the clinical efficacy of treatment and patient prognosis $(4,5,7,26)$.

It is widely understood that the fractionation mode, dosage and site of irradiation affect the pathological process of bone damage after RT $(12,20,27)$. Establishing an irradiation animal model to closely simulate clinical local fractionated RT is invaluable for studying the biological processes of bone structure and bone tissue cells in vivo. To date, animal models of bone injury following RT have predominantly adopted single 2 Gy whole-body irradiation (21), 2 Gy fractionated irradiation several times or a single large (20 Gy) dose administration (28), or 5 Gy large dose fractionated irradiation four times $(11,14)$. These irradiation models are associated with certain defects in the fractionated mode, irradiation dosage, irradiation location and other aspects, failing to truly simulate the clinical RT dose and fractionated mode, or lacking practical applicability due to numerous administrations of anesthetic. At present, the routine practice in the clinical setting is splitting RT, 2 Gy for 30 times or 8 Gy for 5 times with a larger split dose, and these two modes have the same biological equivalent dosage (8). Further considering the difference in relative volume and radiation sensitivity between rats and humans, the application of local irradiation with $8 \mathrm{~Gy}$ for three times can better simulate clinical local fractionated RT and simplify animal experimentation. Therefore, it is widely used in the tumor RT murine and rat models.

Regarding the selection of experimental animals and the exposed location, the local irradiation model of unilateral or bilateral hindlimbs of rodents is commonly adopted $(11,12,14)$. The location of radiation exposure includes the entire lower extremity (femur, tibia and foot), representing $\sim 20 \%$ of the total bone of the body, and is equivalent to the bone ratio of the clinical RT exposed pelvic area, making it more suitable for in vivo studies of bone loss after radiation exposure. In addition, the establishment of the irradiated model of unilateral hindlimb enables the use of the contralateral unexposed hindlimb to observe the systemic effects.

Therefore, there is evidence on the systemic effect in bone loss following single limb irradiation in that bone loss occurs not only at the irradiated site, but at the contralateral and non-irradiated sites as well. Studies on the mechanism of systematic bone loss after local irradiation are crucial for developing prevention and treatment strategies for cancer patients. In the present study, a local irradiation rat model was established to simulate the clinical focal RT procedure and investigate the effects of single 2 Gy local irradiation and 


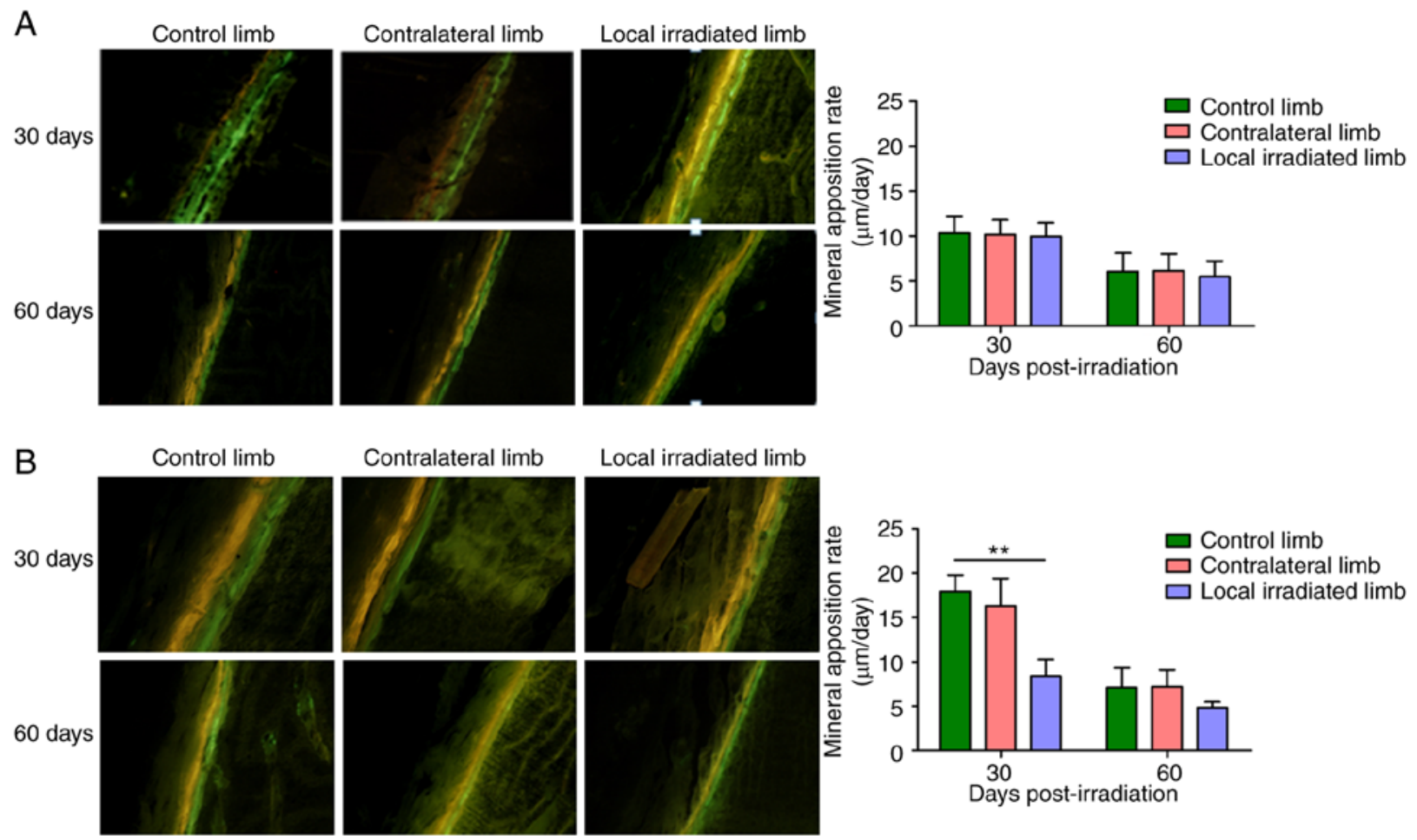

Figure 5. Representative tetracycline hydrochloride and calcein double-labeling in the midshaft and dynamic histomorphometric analysis quantifying mineral apposition rate $(\mu \mathrm{m} / \mathrm{day})$ in the irradiated and contralateral femur relative to control mice at 30 and 60 days post-irradiation. (A) Single 2 Gy local irradiation. (B) 3x8 Gy local irradiation. Magnification, x100. The yellow and green fluorescent lines indicate labeled tetracycline hydrochloride and calcein, respectively. Mineral apposition rate $(\mu \mathrm{m} / \mathrm{day})=$ double fluorescence line spacing/days between the two lines. ${ }^{* *} \mathrm{P}<0.01$ control vs. irradiated limb.
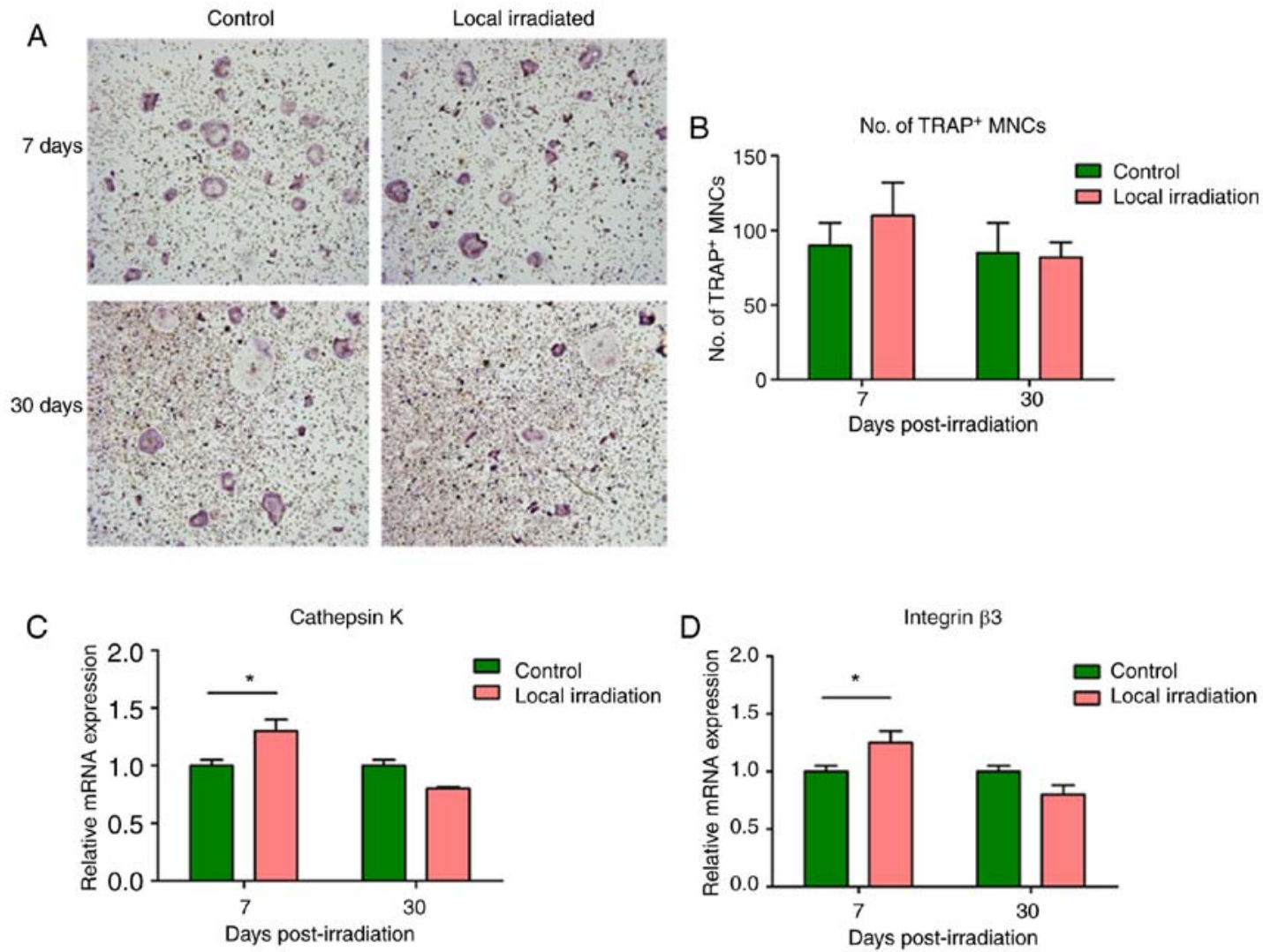

Figure 6. Changes of TRAP-positive cell differentiation from BMMs and marker gene expression following single 2 Gy irradiation. (A) TRAP staining at 7 and 30 days after irradiation (magnification, $\mathrm{x} 40$ ). (B) Number of TRAP-positive MNCs. Expression of the cell differentiation marker genes (C) cathepsin $\mathrm{K}$ and (D) integrin $\beta 3$. The expression levels of cathepsin $\mathrm{K}$ and integrin $\beta 3$ were significantly upregulated at 7 days post-irradiation; however, at 30 days post-irradiation, the expression tended to return to normal levels. "P<0.05. TRAP, tartrate-resistant acid phosphatase; BMMs, bone marrow-derived macrophages; MNCs, mononuclear cells. 
A
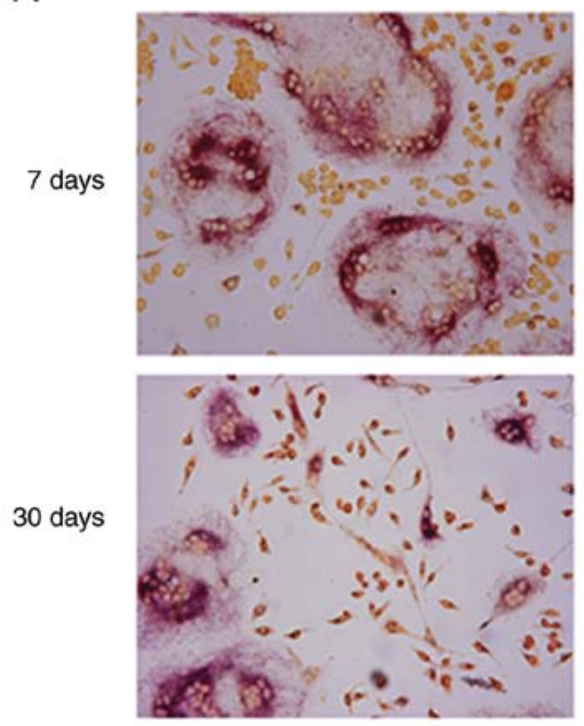

Contralateral limb
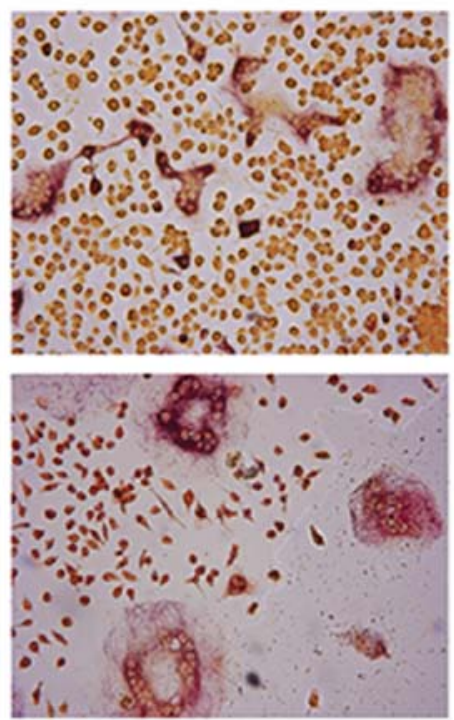
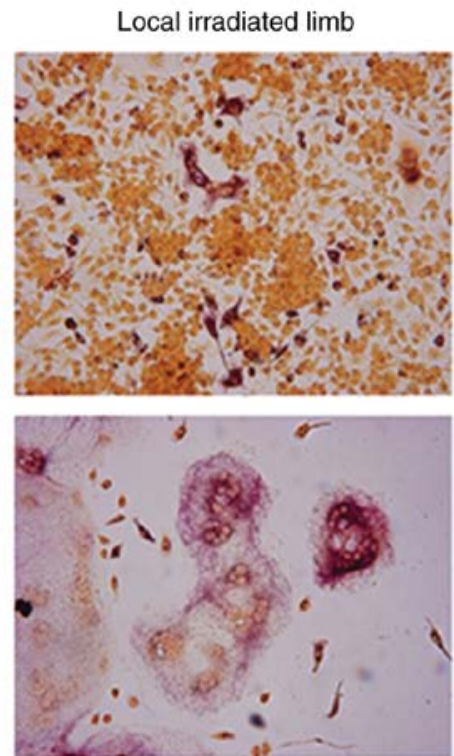

B

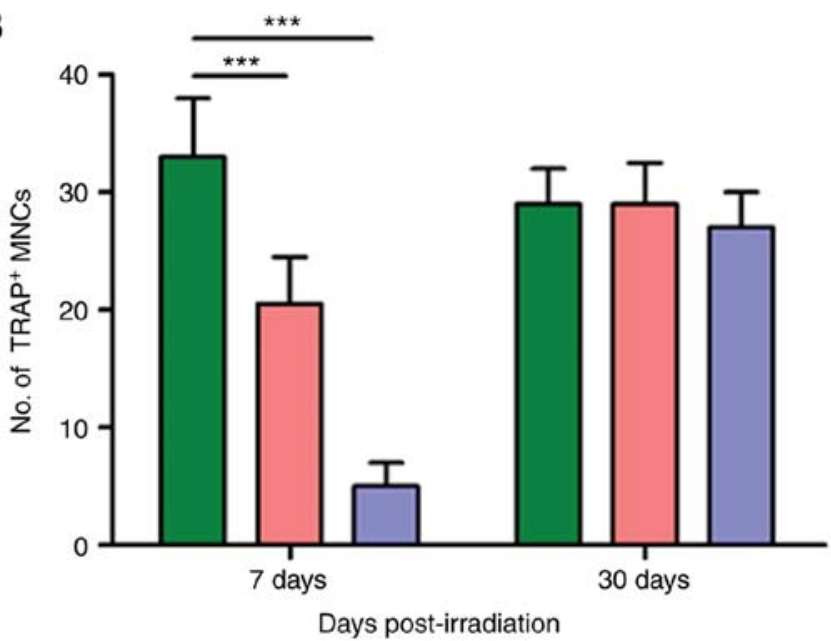

$\square$ Control limb

Contralateral limb

Local irradiated limb

Figure 7. Changes of TRAP-positive cell differentiation from BMMS after 3x8 Gy local irradiation. (A) TRAP staining at 7 and 30 days after irradiation (magnification, x100). (B) Number of TRAP-positive MNCs. At 7 days post-irradiation, the differentiation and fusion of BMMs to form osteoclasts in the local irradiated limb and the contralateral limb were significantly reduced compared with the control limb. While at 30 days post-irradiation, the number of TRAP-positive cells and osteoclastogenesis ability of BMMs returned to normal levels, with no statistically significant difference compared with the control group. $n=6 .{ }^{* * *} \mathrm{P}<0.001$. TRAP, tartrate-resistant acid phosphatase; BMMs, bone marrow-derived macrophages. MNCs, mononuclear cells.

3x8 Gy fractionated local irradiation on bone. The results demonstrated that, at 3 days after single 2 Gy local irradiation, the lumbar spine aBMD exhibited a significant decline, and then gradually recovered. The results of microCT scan revealed that the cortical bone thickness of the directly irradiated femur and the contralateral unexposed femur was significantly lower compared with that of the control group, and the trabecular bone separation and trabecular pattern factor in cancellous bone were significantly increased. These results support that, in the early stage after local irradiation, bone loss and structural damage may occur both in the directly irradiated and the unexposed bone, which is consistent with the changes observed in lumbar vertebrae. The occurrence of early radiation-induced bone injury and its remote effects post-irradiation were confirmed, and such injury was restored in the single 2 Gy local radiation model. Consistent with the clinical report of systemic osteoporosis in cancer patients after radiotherapy, significant bone loss was also observed in sites outside the irradiated area of rats in our experiment. These results demonstrate the applicability of our experimental irradiation-induced bone loss mice model. Vascular injury, inflammation, and production of reactive oxygen species are thought to be the most important factors leading to the deleterious effects of radiation at the distal skeletal and non-skeletal sites. In addition, osteocytes are the most abundant cells in bone tissue and exhibit multiple regulatory effects, while damage of osteocytes may play a key role in the development of systemic bone loss (29). In clinical practice, 2 Gy per time in multiple fractionated radiation is mostly applied, but if the total irradiation dose is significantly higher than 2 Gy, it may result in more serious bone damage that is possibly irreversible. Bone loss and bone structural damage may further lead to osteoporosis and increased risk of pathological fracture $(2,7)$. In clinical focal radiotherapy, the possibility of bone loss and osteoporosis remains high, even in bone tissue that is not directly exposed to radiation, i.e., both the directly irradiated 
site and the non-irradiated bone tissue of patients exhibit an elevated fracture risk after RT $(16,17)$. Therefore, more attention should be paid to the effective prevention of local and systemic bone loss, osteoporosis and fracture in clinical RT patients.

In the present study, a clinical SBRT high-dose fractionated model was simulated, and the left hindlimb was irradiated with three fractionated doses of $8 \mathrm{~Gy}(3 \times 8 \mathrm{~Gy})$. At 3 days (total dose of $8 \mathrm{~Gy}$ ) and 7 days (total dose of $24 \mathrm{~Gy}$; 2 days after the third exposure) after the first exposure, the bone density of the irradiated rats exhibited a rapid decline. At 2 months after the first exposure, the lumbar vertebral density of the irradiated rats remained significantly lower. MicroCT three-dimensional reconstruction demonstrated that irradiation could lead to a reduction in the number of bone trabeculae in the lumbar spine as well as the femur, along with a decrease in trabecular junction points and an increase in the degree of separation. According to the analysis of the bone morphometric parameters, stress bone loss occurred at the early stage of radiation exposure, which was mainly manifested as loss of cancellous bone and destruction of bone structural integrity, including a decrease of BV/TV and Tb.N and an increase of Tb.Sp and Tb.Pf, whereas the bone loss and structural damage persisted over a long period after radiation exposure. In addition, the MAR value decreased, indicating a significant decrease in osteogenic ability. These results indicate that the high-dose fractionated irradiation simulating clinical focal RT exerted early and long-term damage effects on bone tissue, and systemic injury was obvious. Therefore, it is necessary to acknowledge the risk of irreversible bone damage following clinical RT.

Previous, in vivo studies demonstrated that rats locally irradiated with single 2 Gy dose of X-ray exhibit marked bone loss and bone microstructural deterioration. Furthermore, an increased number and enhanced activity of osteoclasts also appears to be an important factor for bone loss after RT (15,30-32). In the present study, at 7 and 30 days post-irradiation, mononuclear cells from the bone marrow of rats were isolated and induced to differentiate into osteoclasts, in order to investigate the effects of ionizing radiation on the osteoclastogenesis potential of BMMs. The results revealed that in the early stages after single 2 Gy dose of X-ray, local irradiation promoted the osteoclast differentiation potential of BMMs, which was manifested by the increased number of TRAP-positive multinuclear cells, and the significantly upregulated mRNA expression levels of cathepsin $\mathrm{K}$ and integrin $\beta 3$, two well-known markers of osteoclastogenesis. However, in the simulated clinical high-dose fractionated model of 3x8 Gy local irradiation, there was an obvious inhibition of osteoclastogenesis potential even in the early stages after irradiation; the number of TRAP-positive multinuclear cells induced by BMMs from the irradiated and the contralateral limb was significantly reduced by 83 and $35 \%$, respectively. It was confirmed that inhibition of osteoclastogenesis potential occurred early in clinical local fractionated RT, and it also confirmed the systemic effect of high-dose irradiation on suppression of bone marrow, which may be caused by excessive inhibition of bone marrow-derived cells during high-dose irradiation. Therefore, unlike the single 2 Gy local irradiation model, high-dose fractionated local irradiation (3x8 Gy) could significantly cause the inhibition of bone marrow at early stage post irradiation, leading to reduction of bone marrow-derived osteoclastogenesis potential, which may play an important role in the development of radiation-induced bone loss. However, the reduction in the number of osteoclasts may be restored to normal levels at the later stages of irradiation. The cellular mechanism of bone injury with different dose patterns requires further investigation $(30,32)$.

In the present study, a biological irradiator with its own beam-limiting collimator and a laboratory-made lead shielding device were used to simulate clinical focal RT irradiation, and a rat model of bone loss caused by local irradiation of unilateral hindlimb was successfully constructed, which may be used to observe the local and systemic bone damage effects caused by ionizing radiation. It has been demonstrated that bone loss and bone structural damage could occur at the early post-irradiation stage, both as local and systemic effects. The bone injury caused by single 2 Gy local radiation is likely to recover completely, however, the bone damage after three $8 \mathrm{~Gy}$ doses of local radiation appears earlier, lasts for a longer period of time and is not easily reversed. There appears to be a different cellular mechanism underlying bone damage in different dosages and fractionation modes $(15,30,31)$, appearing as over-activity of osteoclast function in the early stages of low-dose radiation exposure, while the inhibition of bone marrow-derived cells was the main manifestation in the simulated clinical RT high-dose radiation exposure.

\section{Acknowledgements}

Not applicable.

\section{Funding}

The present study was funded by the Shanghai Natural Science Fund (grant no. 14ZR1401600) and the Shanghai Municipal Commission of Health (grant nos. 2013ZYJB0801 and 20154Y0202).

\section{Availability of data and materials}

The datasets generated and analyzed in the present study are all included in this published article.

\section{Authors' contributions}

GZ designed the study and revised the article. JZ, JC and JW performed the experiments. LT and FH analyzed the data. JZ wrote the manuscript. All authors have read and approved the final version of this manuscript for publication.

\section{Ethics approval and consent to participate}

All the animal experimental procedures were approved by the Committee for Ethical Use of Experimental Animal at Fudan University (Shanghai, China) and the ethical approval registration number for animal study is 201703FYSZGY01.

\section{Patient consent for publication}

Not applicable. 


\section{Competing interests}

The authors declare that they have no competing interests.

\section{References}

1. Bray F, Ferlay J, Soerjomataram I, Siegel RL, Torre LA and Jemal A: Global cancer statistics 2018: GLOBOCAN estimates of incidence and mortality worldwide for 36 cancers in 185 countries. CA Cancer J Clin 68: 394-424, 2018.

2. D'Oronzo S, Stucci S, Tucci M and Silvestris F: Cancer treatment-induced bone loss (CTIBL): Pathogenesis and clinical implications. Cancer Treat Rev 41: 798-808, 2015.

3. Fornetti J, Welm AL and Stewart SA: Understanding the bone in cancer metastasis. J Bone Miner Res 33: 2099-2113, 2018.

4. Baxter NN, Habermann EB, Tepper JE, Durham SB and Virnig BA: Risk of pelvic fractures in older women following pelvic irradiation. JAMA 294: 2587-2593, 2005.

5. Elliott SP, Jarosek SL, Alanee SR, Konety BR, Dusenbery KE and Virnig BA: Three-dimensional external beam radiotherapy for prostate cancer increases the risk of hip fracture. Cancer 117 : 4557-4565, 2011

6. Body JJ, Terpos E, Tombal B, Hadji P, Arif A, Young A, Aapro M and Coleman R: Bone health in the elderly cancer patient: A SIOG position paper. Cancer Treat Rev 51: 46-53, 2016.

7. Handforth C, D'Oronzo S, Coleman R and Brown J: Cancer treatment and bone health. Calcif Tissue Int 102: 251-264, 2018.

8. Willey JS, Lloyd SA, Nelson GA and Bateman TA: Ionizing radiation and bone loss: Space exploration and clinical therapy applications. Clin Rev Bone Miner Metab 9: 54-62, 2011.

9. Sparks RB, Crowe EA, Wong FC, Toohey RE and Siegel JA: Radiation dose distributions in normal tissue adjacent to tumors containing (131)I or (90)Y: The potential for toxicity. J Nucl Med 43: 1110-1114, 2002.

10. Aoki M, Sato M,Hirose K, Akimoto H, Kawaguchi H,Hatayama Y, Ono S and Takai Y: Radiation-induced rib fracture after stereotactic body radiotherapy with a total dose of 54-56 Gy given in 9-7 fractions for patients with peripheral lung tumor: Impact of maximum dose and fraction size. Radiat Oncol 10: 99, 2015.

11. Oest ME, Policastro CG, Mann KA, Zimmerman ND and Damron TA: Longitudinal effects of single hindlimb radiation therapy on bone strength and morphology at local and contralateral sites. J Bone Miner Res 33: 99-112, 2018.

12. Wright LE, Buijs JT, Kim HS, Coats LE, Scheidler AM, John SK, She Y, Murthy S, Ma N, Chin-Sinex HJ, et al: Single-limb irradiation induces local and systemic bone loss in a murine model. J Bone Miner Res 30: 1268-1279, 2015.

13. Zhang J, Zheng L, Wang Z, Pei H, Hu W, Nie J, Shang P, Li B, Hei TK and Zhou G: Lowering iron level protects against bone loss in focally irradiated and contralateral femurs through distinct mechanisms. Bone 120: 50-60, 2019.

14. Oest ME, Franken V, Kuchera T, Strauss J and Damron TA: Long-term loss of osteoclasts and unopposed cortical mineral apposition following limited field irradiation. J Orthop Res 33 334-342, 2015.

15. Sun W, Zhao C, Li Y, Wang L, Nie G, Peng J, Wang A, Zhang P, Tian W, Li Q, et al: Osteoclast-derived microRNA-containing exosomes selectively inhibit osteoblast activity. Cell Discov 2: $16015,2016$.

16. Bazire L, Xu H, Foy JP, Amessis M, Malhaire C, Cao K, De La Rochefordiere A and Kirova YM: Pelvic insufficiency fracture (PIF) incidence in patients treated with intensity-modulated radiation therapy (IMRT) for gynaecological or anal cancer: Single-institution experience and review of the literature. Br J Radiol 90: 20160885, 2017.
17. Uezono H, Tsujino K, Moriki K, Nagano F, Ota Y, Sasaki R and Soejima T: Pelvic insufficiency fracture after definitive radiotherapy for uterine cervical cancer: Retrospective analysis of risk factors. J Radiat Res 54: 1102-1109, 2013.

18. Wernle JD, Damron TA, Allen MJ and Mann KA: Local irradiation alters bone morphology and increases bone fragility in a mouse model. J Biomech 43: 2738-2746, 2010.

19. Rana T, Schultz MA, Freeman ML and Biswas S: Loss of Nrf2 accelerates ionizing radiation-induced bone loss by upregulating RANKL. Free Radic Biol Med 53: 2298-2307, 2012.

20. Hui SK, Fairchild GR, Kidder LS, Sharma M, Bhattacharya M, Jackson S, Le C, Petryk A, Islam MS and Yee D: The influence of therapeutic radiation on the patterns of bone remodeling in ovary-intact and ovariectomized mice. Calcif Tissue Int 92: 372-384, 2013

21. Willey JS, Lloyd SA, Robbins ME, Bourland JD, Smith-Sielicki H, Bowman LC, Norrdin RW and Bateman TA: Early increase in osteoclast number in mice after whole-body irradiation with 2 Gy X rays. Radiat Res 170: 388-392, 2008.

22. Kondo H, Searby ND, Mojarrab R, Phillips J, Alwood J, Yumoto K, Almeida EA, Limoli CL and Globus RK: Total-body irradiation of postpubertal mice with (137)Cs acutely compromises the microarchitecture of cancellous bone and increases osteoclasts. Radiat Res 171: 283-289, 2009.

23. Lucas PA, Aubineau-Lanièce I, Lourenço V, Vermesse D and Cutarella D: Using LiF:Mg,Cu,P TLDs to estimate the absorbed dose to water in liquid water around an 192Ir brachytherapy source. Med Phys 41: 011711, 2014.

24. Fartaria MJ, Reis C, Pereira J, Pereira MF, Cardoso JV, Santos LM, Oliveira C, Holovey V, Pascoal A and Alves JG: Assessment of the mean glandular dose using LiF:Mg,Ti, $\mathrm{LiF}: \mathrm{Mg}, \mathrm{Cu}, \mathrm{P}, \mathrm{Li}_{2} \mathrm{~B}_{4} \mathrm{O}_{7}: \mathrm{Mn}$ and $\mathrm{Li} 2 \mathrm{~B} 4 \mathrm{O} 7: \mathrm{Cu}$ TL detectors in mammography radiation fields. Phys Med Biol 61: 6384-6399, 2016.

25. Livak KJ and Schmittgen TD: Analysis of relative gene expression data using real-time quantitative PCR and the 2(-Delta Delta $\mathrm{C}(\mathrm{T})$ ) method. Methods 25: 402-408, 2001.

26. Bernier J, Hall EJ and Giaccia A: Radiation oncology: A century of achievements. Nat Rev Cancer 4: 737-747, 2004.

27. Jia D, Gaddy D, Suva LJ and Corry PM: Rapid loss of bone mass and strength in mice after abdominal irradiation. Radiat Res 176: 624-635, 2011.

28. Xu X, Li R, Zhou Y, Zou Q, Ding Q, Wang J, Jin W, Hua G and Gao J: Dysregulated systemic lymphocytes affect the balance of osteogenic/adipogenic differentiation of bone mesenchymal stem cells after local irradiation. Stem Cell Res Ther 8: 71, 2017.

29. Bonewald LF: The role of the osteocyte in bone and nonbone disease. Endocrinol Metab Clin North Am 46: 1-18, 2017.

30. Willey JS, Livingston EW, Robbins ME, Bourland JD, Tirado-Lee L, Smith-Sielicki H and Bateman TA: Risedronate prevents early radiation-induced osteoporosis in mice at multiple skeletal locations. Bone 46: 101-111, 2010.

31. Alwood JS, Shahnazari M, Chicana B, Schreurs AS, Kumar A, Bartolini A, Shirazi-Fard Y and Globus RK: Ionizing radiation stimulates expression of pro-osteoclastogenic genes in marrow and skeletal tissue. J Interferon Cytokine Res 35: 480-487, 2015.

32. Ren L, Song ZJ, Cai QW, Chen RX, Zou Y, Fu Q and Ma YY: Adipose mesenchymal stem cell-derived exosomes ameliorate hypoxia/serum deprivation-induced osteocyte apoptosis and osteocyte-mediated osteoclastogenesis in vitro. Biochem Biophys Res Commun 508: 138-144, 2019.

This work is licensed under a Creative Commons Attribution-NonCommercial-NoDerivatives 4.0 International (CC BY-NC-ND 4.0) License. 